swelling in the subglottic space subsided after about two weeks, though the result of the electrolysis was insignificant, but a small slough coming away. I had expected a better result as a similar current in the skin would have produced a large slough. A second attempt with the electrolytic needle did not add much to the result, though the lumen left in the larynx by the subsidence of the swelling was undoubtedly larger than before the operation. As the growth on the posterior wall of the trachea seemed responsible for the greater part of the dyspnea, I succeeded in getting my patient's consent to remove as much of this as I could through the tracheotomy wound. By means of the wire snare and a pair of Fränkel's forceps it was possible to take away the greater part of the intratracheal swelling, the most prominent part of which was situated at the second and third tracheal ring; this, when seen under the microscope, proved to be typical thyroid tissue. I repeatedly seized the swellings in the subglottic region with Scheinmann's cutting laryngeal forceps, from above, but the great firmness of the tissue made it impossible to remove even the smallest piece. After this the patient went about with her tracheotomy tube, as her experience with suffocation made her feel unsafe without it, for though her breathing could be carried on quite well without it, she feared another attack of swelling and suffocation. She still refused operative measures from without, so $I$ attempted to reduce the subglottic swelling with the galvanocautery, and, though I succeeded in introducing a cautery electrode into the growth from above, the result was infinitesimal. It is difficult to use instruments with accuracy in the subglottic region, and the necessity of avoiding injury to the vocal cords made it possible to burn only a very little at a time. The patient finally left me, improved but not daring to take out her tracheotomy-tube. I. heard of her through others whom she consulted, and presume that perhaps by this time she has consented to a laryngo-tracheotomy, the only operative measure of value in this condition, as the thyroid tissue can only be removed by being dissected out.

I hope that my description will lead to the recognition of other cases of this kind which may be less rare than supposed. The fact that my patient had no appreciable thyroid gland in her neck leads to the supposition that a great part of it at least was represented by the extensive amount in her larynx and trachea, an amount rrcater than in most of the cases heretofore reported. Though in the other cases the thyroid tissue is described as forming a tumor, in mine its diffuseness made such a term inappropriate, it being rather a thick lining for the entire cricoid circumference and extending in the same manner down the back of the trachea. The only tumorlike formation was the prominence on the back of this that was removed.

In a recent article myxedema is described as following the removal of thyroid tissue at the base of the tongue, so it seems possible that the thyroid tissue in unusual situations may perhaps take the place of the normal gland. In regard to the power possessed by thyroid elements to penetrate and grow in other structures than the proper capsule of the thyroid gland, Dr. Christian Fenger, in a personal communication, mentioned a case in which the thyroid tissue had penetrated the lumen of the jugular vein. Dr. Fenger regards the microscopic specimens obtained from my case as typical thyroid tissue.

My experience with electrolysis in the subglottic region would make me hesitate to use it there again unless the patient had had a previous tracheotomy. A bipolar electrode, if it could be introduced, would doubtless be better than the monopolar one employed by me. Electrolysis would be an excellent method to employ for ecchondroma in this difficult region. On account of its loose areolar tissue the hypoglottic region is much more apt to react after irritation by inflammatory swelling than the larynx proper. The connective tissue, in the specimens presented, forms a much denser network than that of the normal thyroid gland, a fact which accounts for the great firmness presented by the growth.

288 E. Huron Street.

\section{OPEN TREATMENT OF SUPPURATION OF THE KNEE-JOINT.*}

W. J. MAYO, A.M., M.D.

Surgeon to St. Mary's Hospital; Surgeon to C. \& N.-W. Ry. Co. ROCHESTER, MINN.

In the January, 1895, issue of the Annals of Surgery, Dr. C. H. Mayo reported a case of acute suppuration of the knee-joint, which he had treated July 30, 1894, by a transverse incision across the front of the joint in the same manner as for excision. This enables the surgeon to get at every part of the joint, converting its many recesses into a simple open cavity. The result of the operation is astonishing. In a few hours a patient in whom an amputation seemed to be the only hope is on the road to recovery; such a procedure gives all the benefits of the mutilating operation without shock or injury to important structures. It must be understood that this method of treatment is for the more advanced cases after the usual measures have been exhausted and amputation appears to be necessary. In most instances the capsule has been perforated and the soft parts above and below the joint are infiltrated with septic material. All of the important structures are in the popliteal space, and a transverse incision through the patella enables the surgeon to pack the joint with gauze, and treat it as an open wound. By going through the patella, secondary sutures are given a firm support after the infection is under control. The abseesses, if they exist in the soft parts, are freely opened and drained. The margins of the wound granulate rapidly and in from two to three weeks, when the discharge has practically ceased, the cut patella is reunited; the excess of granulations removed and the soft parts allowed to granulate.

Surprising to relate, not all of the cases are followed by ankylosis. If the cartilage of the joint is not eroded considerable motion may be ultimately obtained, although this is a secondary consideration, as the condition is one which threatens life or limb. The reason ankylosis does not always follow is because the adhesions are developed in the same manner as a pannus projects over the cornea and, unless the cartilage is completely covered by the advancing granulation, the portion which remains smooth permits of motion. If erosion of the cartilage has taken place ankylosis will necessarily result no matter what method of treatment is followed.

For the milder cases in which the capsule is intact irrigation with antiseptic solutions or with pure carbolic acid followed by alcohol, as advocated by Phelps, is often all that is necessary. If this fails, lateral incisions, as practiced by Halsted, or tubular drains after the plan of Edmund Andrews, may be sufficient, but there are still a number of neglected cases, especially punctured wounds, in which these ordinarily satisfactorv methods are entirely inadequate and the patients have in the past come to amputation or death. Open treatment by transverse incision across the entire front and lateral aspects of the joint gives all of the benefits of amputation without its risks, and saves for the victim a most useful limb. We have practiced this method for six years in a number of cases with the most satisfactory results, and in chil-

* Read at the annual meeting of the American Academy of Railway Surgeons, at the meeting held in St. Paul, Minn., Sept 5 and 6,1900 . 
dren have obtained a range of motion from 15 to 60 per cent. of the normal. In conclusion I can not do better than to cite Dr. Arpad Gerster, ${ }^{1}$ who says that Dr. C. H. Mayo's suggestion struck him as very plausible, and he has "applied it in two cases. The result has been excellent. As all know, these acute forms of joint phlegmon necessarily end in ankylosis, if, indeed, they do not cause loss of limb or life. The cartilaginous covering of the bones forming the joint is destroyed and, therefore, ankylosis is inevitable. Knowing this fact, the surgeon in adopting this method of treatment frankly accepts the necessity for ankylosis. The road then becomes very clear. The treatment consists in laying the joint open by a transverse incision, extending from one condyle to the other, just as a joint is laid open for resection. Then as many more incisions are made as may be necessary, in order to open up every collection of pus or fluid in or about the joint. The capsule in all of these cases is very intumescent and swollen and for this reason occludes all drainage tubes in a way to make retention inevitable. Then lay the joint freely open, removing or not removing tissues, as the case may be; pack and drain every recess, and treat it as an open wound. It is astonishing how rapidly the phlegmonous process ceases when compared with other methods of treatment. After the symptoms of active inflammation have subsided, the joint is put in proper position and ankylosis is allowed to take place."

Since these remarks by Dr. Gerster, this method of operation has been applied in a large number of cases at Mt. Sinai Hospital, New York City, with the most satis factory results.

QUANTITATIVE TESTS FOR PROTEOLYSIS.*

$$
\text { A. I. BENEDICT, A.M., M.D. }
$$

Professor of Physiology and Digestive Diseases, Dental Department, University of Buffalo; Editor American Therapist.

BUFFALO, N. $\mathbf{Y}$.

(Concluded from p. 808.)

The first table herewith presented gives the results of investigations on students who were free from subjective and objective evidences of stomach trouble, except for some transient disturbances of function as noted. The test meal throughout this series consisted of 50 grams of dry bread, 5 grams of butter. and 250 cubic centimeters of cold water, taken as a noon meal and removed by siphonage from sixty to ninety minutes after the completion of the meal. In no instance were remnants of breakfast found. In eleven of the sixteen examinations, syntonin was either altogether absent or present in the merest trace. The maximum precipital. was $20 / 100$ c.c. in 10 c.c. As the qualitative examinations showed some slight variations from the theoretic normal, it was with some surprise that the following facts were deduced: 1 . The presence of a measurable quantity of syntonin, was not connected with a failure of free $\mathrm{HCl}$. In every one of the instances showing excess of syntonin, $\mathrm{HCl}$ was present by the resorcin and cane-sugar test. Conversely, $\mathrm{HCl}$ had all been fixed in three of the cases which were most typical so far as amount of syntonin was concerned. Hence, no generalization could be made as to the relation between proteolysis, at this stage, and presence of free HCl. 2. The excess of syntonin was connected with no conspicuous failure of ferments. However, it must be

1. Tran. of N. Y. Surg. Soc., xxil, p. 503.

* Awarded First Gold Medal in Medicine, by the Prize Commit tee of the American Medical Association, at the Fifty-first an nual Meeting, held at Atlantic City, N. J., June 5-8, 1900. admitted that no attempt was made to carry out a test of ferments at every examination and that our tests for ferments are exceedingly crude and unsatisfactory. 3 . The excess of syntonin was not conmected with an excess of lactic acid. The test employed was that with ferric chlorid masked by gentian violet, as recommended by Hewes, of Boston, and others. This test seems just delicate enough to detect an excess of lactates without reacting to the small proportion normally present after ordinary test-meals. 4. The excess of syntonin was not connected with an abnormality in carbohydrate digestion. 5. The excess of syntonin was not in relation with the amount of chyme present at the time of extraction. 6. The excess of syntonin was not due to the time at which the stomach was emptied, within the limits of sixty and ninety minutes after the meal. 7 . Of the five occurrences of a measurable amount of syntonin four were in the case of one subject.

'Three cases showed albumose only in traces, the remainder varied between $5 / 100$ and $20 / 100$, eight yielding almost exactly $10 / 100$ c.c. in 10 c.c. of filtered chyme. This variation seemed entirely within physiologic limits and without obvious cause.

Only nine of the series were treated with phosphomolybdic acid, the earlier examinations showing only the inapplicability of tannin and unmodified mercuric salts. One of the nine cases yielded a precipitate of peptone amounting only to $40 / 100$ c.c. in 10 c.c. of filtered chyme, but the stomach contents could not be extracted until after dilution. The remaining eight cases varied between 110/100 and 290/100. Careful study failed to explain this wide variation. Disregarding the sample obtained after dilution, the maximum and the minimum of peptone were found in stomach contents which corresponded almost perfectly in other respects. Several control tests showed that, in the absence of starch, the precipitate of peptone with phosphomolybdic acid is almost exactly six times as bulky as that obtained with tannin. Calculating with this coefficient, three more cases may be added to the eight and the conclusions drawn are corroborative of the other results, namely, that the peptone precipitate is normally between onetenth and three-tenths of the volume of the filtered chyme, if phosphomolybdic acid is employed.

In regard to other tests carried out in conjunction with the proteolysis, one or two observations should be made. The apparent absurdity of noting an absence of frce acids in general while free $\mathrm{HCl}$ in particular is present is explained by the different sensitiveness of the respective tests. Benzopurpurin, according to the writer's investigation, is the most sensitive of the aniline dyes for general free acidity. Next in order comes Congo-red. The others thus far employed are so far inferior either in delicacy or in distinctiveness of color change, that they need not be considered. The series of observations on normal individuals corroborates the conclusions reached by Hewes, of Boston, based on a similar but larger series, namely, that the small bulk of chyme assigned by German writers as normal, after the test breakfast. does not hold good for Americans. ${ }^{1}$ The totals obtained by the writer varied from 35 to 200 c.c., the average being 83 , at least double the amount formally taught as normal. Although every precaution was used to secure the thorough emptying of the stomachs, it is obvious that any possible error must be in the direction of minimizing the bulk of the contents.

While a larger series of observations will be necessary to determine details, especially as to the proportions of

1. Boston Medical and Surgical Journal, 1897. 